\title{
Separator-less Amino Acid-powered Biofuel Cell Through an Artificial Multi-enzyme Cascade Reaction
}

\section{T Satomura ( $\sim$ satomura@u-fukui.ac.jp )}

University of Fukui: Fukui Daigaku https://orcid.org/0000-0003-0151-9691

\section{Kousaku Horinaga}

University of Fukui: Fukui Daigaku

\section{Eiichiro Takamura}

University of Fukui: Fukui Daigaku

\section{Hiroaki Sakamoto}

University of Fukui: Fukui Daigaku

\section{Haruhiko Sakuraba}

Kagawa University: Kagawa Daigaku

\section{Toshihisa Ohshima}

Osaka Institute of Technology: Osaka Kogyo Daigaku

\section{Shin-ichiro Suye}

University of Fukui: Fukui Daigaku

\section{Research Article}

Keywords: Separator-less enzymatic biofuel cell, thermophilic enzyme, enzyme cascade, polymer-bound redox mediator

Posted Date: October 22nd, 2021

DOI: https://doi.org/10.21203/rs.3.rs-976016/v1

License: (c) (1) This work is licensed under a Creative Commons Attribution 4.0 International License. Read Full License 
$1 \quad$ Biotechnology letters

2 Original Research Paper: Microbial and Enzyme Technology

3

4 Title: Separator-less amino acid-powered biofuel cell through an artificial multi-enzyme cascade

$5 \quad$ reaction

6 Takenori Satomura ${ }^{\text {a,b*}}$, Kousaku Horinaga ${ }^{c}$, Eiichiro Takamura ${ }^{a}$, Hiroaki Sakamoto ${ }^{\text {a }}$, Haruhiko

$7 \quad$ Sakuraba $^{\mathrm{d}}$, Toshihisa Ohshima ${ }^{\mathrm{e}}$, Shin-ichiro Suye $\mathrm{e}^{\mathrm{a}}$

8 a Division of Engineering, Faculty of Engineering, University of Fukui, 3-9-1 Bunkyo, Fukui 910-

9 8507, Japan

10 b Life Science Innovation Center, University of Fukui, 3-9-1 Bunkyo, Fukui 910-8507, Japan

$11{ }^{\mathrm{c}}$ Department of Applied Chemistry and Biotechnology, Graduate School of Engineering, University

12 of Fukui, 3-9-1 Bunkyo, Fukui 910-8507, Japan

13 d Department of Applied Biological Science, Faculty of Agriculture, Kagawa University, 2393 Ikenobe,

14 Miki-cho, Kita-gun, Kagawa 761-0795, Japan

$15{ }^{\mathrm{e}}$ Department of Biomedical Engineering, Faculty of Engineering, Osaka Institute of Technology, 5-

16 16-1 Ohmiya, Asahi-ku, Osaka, 535-8585 Japan.

17 *Corresponding author: Takenori Satomura, ORCID: 0000-0003-0151-9691

18 Tel: +81-776-27-8642, Fax:+81-776-27-8747, E-mail: satomura@u-fukui.ac.jp 


\section{Abstract}

21 This study was aimed at constructing a highly stable one-compartment enzymatic biofuel cell (EFC)

without a separator through a multi-enzyme cascade reaction pathway.

23 Results

24 A separator-less EFC composed of a multi-enzyme cascade anode containing four dehydrogenases

from a thermophile and a cathode devised using a multi-copper oxidase mutant with enhanced enzyme

26 activity from a hyperthermophile was developed. To fabricate an EFC without a separator, redox

27 mediators utilized in the enzymatic cascade reaction were also immobilized on the anode. In the

presence of the fuel $100 \mathrm{mM}$ L-proline, the separator-less EFC with four thermophilic dehydrogenase-

modified anode achieved a maximum power density of $11.3 \mu \mathrm{W} / \mathrm{cm}^{2}$ at $37^{\circ} \mathrm{C}$, which was 1.6 -fold

30 higher than that of a similar EFC fabricated with a one enzyme-modified anode. The separator-less

31 EFC composed of a multi-enzyme modified anode maintained approximately $57 \%$ of the load current 
34 Efficient L-proline electric generation utilizing a separator-less EFC composed of a multi-enzyme modified anode through a multi-step fuel oxidation reaction and a highly stable multi-copper oxidase mutant-modified cathode was successfully achieved over a long period. The long-term stability of the separator-less EFC can facilitate its application as an efficient power source for implantable medical devices requiring continuous operation. 
50 Enzymatic biofuel cell (EFC) is an electrochemical device that uses oxidoreductase as an 51 electrocatalyst and converts the chemical energy of its substrate into electric energy. EFCs have high potential for application in implantable medical devices that can generate electricity without added toxicity concerns, unlike traditional batteries such as lithium batteries (Cosnier et al. 2014). However, the practical applications of EFCs are still limited because of their low battery life due to enzyme instability. To address this issue, we have developed EFCs using oxidoreductases from thermophiles as a specific electrode element. Enzymes from thermophiles are known to be considerably more stable at room temperature and across a wide range of $\mathrm{pH}$ than their mesophilic counterparts (Ohshima and Soda 1989; Adams 1993; Li et al. 2005). We have already succeeded in constructing a novel highefficiency bioanode for the multi-step oxidation of L-proline by an artificial electro-metabolic pathway using thermophilic enzymes (Fig. 1) (Satomura et al. 2019). The novel electrode performing multistep oxidation using L-proline as a fuel comprised four kinds of thermophilic dehydrogenases: dyelinked L-proline dehydrogenase from Aeropyrum pernix (ApLPDH), NAD-dependent $\Delta$ 1-pyrroline-5carboxylic acid dehydrogenase from Thermus thermophilus (ThP5CDH), NAD-dependent glutamate dehydrogenase from Pyrobaculum islandicum (PiGDH), and dye-linked NADH dehydrogenase from Geabacillus kaustphilus (GkNADHDH). The electrode modified with these 4 enzymes exhibited an approximately 4.2-fold higher current response than the electrode modified with ApLPDH alone. 
Moreover, the current response of the multi-enzyme modified electrode remained at more than $50 \%$

of its initial current response even after 15 days at room temperature. In addition to the development of a highly efficient anode, we have successfully constructed a highly stable multi-copper oxidase mutant from the hyperthermophilic archaeaon, P. aerophilum, with enhanced electrocatalytic activity (eMcoP) as an element for cathode (Satomura et al. 2021). In this study, we aimed to construct a highly efficient EFC with the multi-enzyme modified electrode as the anode and the eMcoP-modified electrode as the cathode.

In several cases of EFCs, redox mediators are required to shuttle electrons between the enzymes and electrodes. In addition, the anode used in this study requires redox mediators to perform a multienzyme cascade reaction on the electrode. Free redox mediators are known to cause serious crossover reactions with electrodes in the EFC (Katz et al. 1999; Tujimura et al., 2001; Tujimura et al. 2002; Kamitaka et al. 2007). Redox mediators required for the anodic reaction react at the cathode, leading to a decrease in the power of the EFC. In general, to avoid the crossover reactions, separators such as Nafion membranes are incorporated into the EFC; however, these modifications increase the structural complexity if the EFC.

Therefore, we investigated the conditions for immobilizing the redox mediators to the anode and developed a simplified one-compartment EFC without a separator. The use of immobilized redox mediators together with the multi-enzyme cascade based electrodes was aimed at building simple and 
Materials and methods

89

Materials

90

L-Proline and Nafion perfluorinated resin solution were obtained from Sigma-Aldrich (St. Louis, MO,

91

USA). NAD ${ }^{+}$was purchased from Oriental Yeast (Tokyo, Japan). Carbon paper (CP, TGP-H-120) was

purchased from Toray (Tokyo, Japan). 1-Aminopyrene $\left(\mathrm{Py}-\mathrm{NH}_{2}\right)$, multi-walled carbon nanotubes

(MWCNT, 10-20 $\mathrm{nm}$ in diameter and 5-15 $\mathrm{mm}$ in length), 1-(3-dimethylaminopropyl)-3-

ethylcarbodiimide hydrochloride (EDC), $N$-hydroxysuccinimide (NHS), and aminoferrocene were purchased from Tokyo Chemical Industry (Tokyo, Japan). Sodium alginate was obtained from

Nacalai Tesque (Kyoto, Japan). $N$-methylpyrrolidone (NMP) was purchased from FUJIFILM Wako

Pure Chemical Corporation (Osaka, Japan). All other chemicals were of reagent grade.

\section{Preparation of bioanode.}

100 The enzymes composing the artificial enzyme cascade for the bioanode were prepared as described

101 previously (Satomura et al. 2019). Polymer-bound NAD ${ }^{+}$(Alg-NAD) with alginic acid was prepared

102 as previously described (Nakamura et al. 1996). To prepare the polymer-bound ferrocene (Alg-Fc), 20 
111 Subsequently, the electrode was rinsed with NMP and water to prepare the CP/MWCNT/Py- $\mathrm{NH}_{2}$ electrode. An aliquot of $200 \mu \mathrm{L}$ from a solution containing $0.5 \mathrm{mg}$ Alg-NAD $/ \mathrm{mL}, 0.5 \mathrm{mg}$ Alg-Fc $/ \mathrm{mL}$, $100 \mathrm{mM}$ EDC, $100 \mathrm{mM}$ NHS, and $50 \mathrm{mM}$ PKB (pH 7.2) was dropped onto the CP/CNT/Py-NH 2 electrode. The electrode was incubated for $6 \mathrm{~h}$ at $30^{\circ} \mathrm{C}$ and then rinsed with $50 \mathrm{mM} \mathrm{PKB} \mathrm{(pH} \mathrm{7.2)} \mathrm{to}$ prepare the $\mathrm{CP} / \mathrm{CNT} / \mathrm{Py}-\mathrm{NH}(-\mathrm{Alg}-\mathrm{Fc} / \mathrm{Alg}-\mathrm{NAD})$ electrode. The enzyme solution containing onto the $\mathrm{CP} / \mathrm{CNT} / \mathrm{Py}-\mathrm{NH}_{2}$ (-Alg-Fc/-Alg-NAD) electrode. After drying, Nafion solution (20 $\mu \mathrm{L}$ of 
122 The eMcoP for the biocathode was prepared as described elsewhere (Satomura et al. 2021); after

123 soaking the $\mathrm{CP} / \mathrm{CNT}$ electrode in $20 \%(\mathrm{v} / \mathrm{v})$ ethanol, $20 \mu \mathrm{L}$ eMcoP $(0.01 \mu \mathrm{mol} / \mathrm{min})$ was dropped

124 onto the electrode. The electrode was dried at room temperature and then cross-linked in $25 \%$

125 glutaraldehyde vapor for $30 \mathrm{~min}$. After washing the electrode with $10 \mathrm{mM}$ Tris- $\mathrm{HCl}(\mathrm{pH} 7.2)$, the cathode was stored at $4^{\circ} \mathrm{C}$ in $100 \mathrm{mM} \mathrm{PKB} \mathrm{(pH} \mathrm{7.0)} \mathrm{until} \mathrm{use.}$

\section{Electrochemical measurements}

PKB (200 mM, pH 7.2) was used as the electrolyte for all electrochemical measurements. Cyclic analyzer (Model 1205 B, BAS Inc., Tokyo, Japan). The enzyme-modified electrode, platinum wire and $\mathrm{Ag} / \mathrm{AgCl}$ electrode were used as the working, counter and reference electrodes, respectively. The

$\mathrm{CV}$ measurements were recorded at $10 \mathrm{mV} / \mathrm{s}$.

$37^{\circ} \mathrm{C}$ using an ALS electrochemical analyzer (Model $1205 \mathrm{~B}$ ).

136 Evaluation of the long-term stability of EFC was performed using the Bio Battery Tester Model 1703

137 (Keisokuki Center, Osaka, Japan). The voltage of the biofuel cell was adjusted to $0.3 \mathrm{~V}$, and the 138 temporal changes in the value of the load current of the EFC were measured. 


\section{Electrochemical evaluation of polymer-bound redox mediator-immobilized electrode}

141 In our previous study, the efficient oxidation of L-proline to 2-oxogultarate by multi-enzyme cascade

142 reactions was successfully achieved on our designed electrode (Fig. 1) (Satomura et al. 2019). The

143 multi-enzyme cascade reaction was achieved using four thermophilic dehydrogenases (ApLPDH,

144 ThP5CDH, PisGDH, and GKNADHDH) in the presence of two kinds of free redox mediators (NAD ${ }^{+}$

145 and ferrocene). In this study, to construct a separator-less EFC, we immobilized both $\mathrm{NAD}^{+}$and

146 ferrocene on the electrode. First, NAD ${ }^{+}$or aminoferrocene was covalently modified at the carboxylic

147 group of alginic acid using EDC and NHS to generate Alg-NAD and Alg-Fc, respectively (Fig. 1).

148 Next, Py- $\mathrm{NH}_{2}$ was immobilized on the $\mathrm{CP} / \mathrm{CNT}$ electrode. Our previous study revealed that pyrene

149 derivatives containing Py-NH 2 specifically interact with CNTs by $\pi-\pi$ stacking (Sakamoto et al. 2019).

150 After the immobilization of $\mathrm{Py}-\mathrm{NH}_{2}$ on the $\mathrm{CP} / \mathrm{CNT}$ electrode, the amino group in $\mathrm{Py}-\mathrm{NH}_{2}$ and the

151 non-modified carboxylic groups in Alg-NAD and Alg-Fc were conjugated using NHS and EDC to generate a $\mathrm{CP} / \mathrm{CNT} / \mathrm{Py}-\mathrm{NH}_{2}$ (-Alg-Fc/-Alg-NAD) electrode. To investigate the function of the immobilized redox mediators on the electrode, the $\mathrm{CV}$ of the $\mathrm{CP} / \mathrm{CNT} / \mathrm{Py}-\mathrm{NH}_{2}(-\mathrm{Alg}-\mathrm{Fc} / \mathrm{Alg}-\mathrm{NAD})$ electrode modified with four thermophilic enzymes was measured without redox mediators in the 

oxidation of L-proline through a multi-enzyme cascade pathway. [INSERT Fig. 2]

\section{Evaluation of EFC through the multi-step enzymatic L-proline oxidation pathway}

The separator-less EFCs were constructed with an eMcoP-modified cathode and three different thermophilic dehydrogenase-modified anodes (ApLPDH, ApLPDH/P5CDH/GkNADHDH or ApLPDH/P5CDH/PisGDH/GkNADHDH) as the electrodes (Fig. 1), and their electrochemical characteristics were evaluated. The maximum power densities of one-compartment EFCs without separator in the presence of $100 \mathrm{mM}$ L-proline using ApLPDH, ApLPDH/P5CDH/GkNADHDH and $\mu \mathrm{W} / \mathrm{cm}^{2}$ at $0.21 \mathrm{~V}$, and $11.2 \mu \mathrm{W} / \mathrm{cm}^{2}$ at $0.22 \mathrm{~V}$, respectively (Fig. 3). These results clearly show that the power density of the EFC increases with an increase in the number of thermophilic dehydrogenases, and the multi-enzyme cascade reaction occurs efficiently on the electron mediator immobilized anode.

[INSERT Fig. 3] In addition, they indicate that the separator-less EFC composed of anode modified with four dehydrogenases and an eMcoP-modified cathode can generate the highest power output among all EFCs tested in this study. Several separator-less EFCs have been reported so far (Tsujimura 
175 involved only single-step oxidation of the fuel. To our knowledge, this is the first successful example

176 of a separator-less EFC through a multi-enzymatic cascade reaction.

177 The long-term stability of the EFC composed of four thermophilic dehydrogenase-immobilized anode

178 and eMcoP cathode was evaluated at $37^{\circ} \mathrm{C}$ by monitoring the relative load current at $0.3 \mathrm{~V}$. The EFC

179 retained $62 \%$ of the initial load current after continuous operation for 2 days, and $57 \%$ of the initial

180 load current even after 4 days (Fig. 4). [INSERT Fig. 4]

181 Separator-less EFCs can be miniaturized owing to their structural simplicity. Therefore, a high-

182 powered separator-less EFC with long life shows high potential for application as a power source for

183 implantable medical devices such as implanted drug-delivering pumps for the management of chronic

184 pain, cerebral palsy, and diabetes (Barton et al. 2004). Further studies are currently underway to realize

185 the practical biomedical application of such a power source.

\section{Conclusions}

189 A novel separator-less EFC was fabricated through a multi-enzyme cascade pathway using

190 thermophilic enzymes. The maximum power density of the EFC composed of the four dehydrogenase-

191 immobilized anode was 1.6-fold higher than that of the EFC composed of the anode immobilized with

192 ApLPDH alone. Furthermore, the separator-less EFC retained more than 50\% of its initial load current 
193 at $0.3 \mathrm{~V}$ even after continuous operation for 4 days at $37^{\circ} \mathrm{C}$. The high-performance separator-less EFC

194 with a long life exhibits a high potential utility as power source for implantable bio-devices.

195

\section{Declarations}

$197 \quad$ Funding

198 This work was supported by the research grants from the Research and Education Program for Life

199 Science, University of Fukui.

200

201 Conflict of Interest

202 The authors declare that they have no competing interests.

203

204 Data and material availability

205 The datasets generated during and/or analysed during the current study are available from the

206 corresponding author on reasonable request.

207

208 Authors' contributions

209 All authors contributed to the study conception and design. Material preparation, data collection and

210 analysis were performed by Kousaku Horinaga, and Takenori Satomura. The first draft of the 
211 manuscript was written by Takenori Satomura and all authors commented on previous versions of the

212 manuscript. All authors read and approved the final manuscript.

213

\section{References}

215 Adams MW (1993) Enzymes and proteins from organisms that grow near and above 100 degrees C.

$216 \quad$ Annu Rev Microbiol 47:627-658

217 Barton SC, Gallaway J, Atanassov P (2004) Enzymatic biofuel cells for implantable and microscale devices Chem Rev 104:4867-4886

Bellino MG, Soler-Illia GJ (2014) Nano-designed enzyme-functionalized hierarchical metal-oxide mesoporous thin films: en route to versatile biofuel cells. Small 10: 2834-9, 2743

Coman V, Vaz-Domínguez C, Ludwig R, et al. (2008) A membrane-, mediator-, cofactor-less glucose/oxygen biofuel cell. Phys Chem Phys 10: 6093-6096

Kamitaka Y, Tsujimura S, Setoyama N, et al. (2007) Fructose/dioxygen biofuel cell based on direct electron transfer-type bioelectrocatalysis. Phys Chem Phys 9: 1793-1801

Katz E, Willner I, Kotlyar AB (1999) A non-compartmentalized glucose $\mid \mathrm{O}_{2}$ biofuel cell by bioengineered electrode surfaces J Electroanal Chem 479: 64-68 
Ohshima T, Soda K (1989) Thermostable amino acid dehydrogenases: applications and gene cloning. Trends Biotechnol 7:210-214

Sakamoto H, Koto A, Takamura E, et al. (2019) Development of biofuel cell using a complex of highly derivative. J Nanosci Nanotechnol 19: 3551-3557

Satomura T, Horinaga K, Tanaka S, et al. (2019) Construction of a novel bioanode for amino acid powered fuel cells through an artificial enzyme cascade pathway. Biotechnol Lett 41: 605-611

Satomura T, Hirano T, Inagaki K, et al. (2021) Activity enhancement of multicopper oxidase from a hyperthermophile via directed evolution, and its application as the element of a high performance biocathode. J Biotechnol 325: 226-232

Tsujimura S, Fujita M, Tatsumi H, et al. (2001) Bioelectrocatalysis-based dihydrogen/dioxygen fuel cell operating at physiological pH. Phys Chem Phys 3: 1331-1335

Tsujimura S, Kano K, Ikeda T (2002) Glucose/ $\mathrm{O}_{2}$ biofuel cell operating at physiological conditions. 
247 Fig. 1 Schematic illustration of separator-less biofuel cell operated through a multi-enzyme cascade

248 reaction. ApLPDH, dye-linked L-proline dehydrogenase from Aeropyrum pernix; TthP5CDH, NAD -

249 dependent $\Delta^{1}$-pyrrorine-5-carboxylate dehydrogenase from Thermus thermophilus; PiGDH, NAD-

250 dependent L-glutamate dehydrogenase from Pyrobaculum islandicum; GkNADHDH, dye-linked

251 NADH dehydrogenase from Geobacillus kaustophilus; eMcoP, multi-copper oxidase mutant from $P$.

252 aerophilum; P5C, $\Delta^{1}$-pyrrorine-5-carboxylate; Alg-Fc, polymer-bound ferrocene; Alg-NAD, polymer-

253 bound $\mathrm{NAD}^{+}$; $\mathrm{Pyr}_{-\mathrm{NH}_{2}}, 1$-aminopyrene

254

255 Fig. 2 Cyclic voltammetry curves of ApLPDH/ThP5CDH/PisGDH/GkNADHDH-modified

$\mathrm{CP} / \mathrm{CNT} / \mathrm{Py}-\mathrm{NH}_{2}(-\mathrm{Alg}-\mathrm{Fc} / \mathrm{Alg}-\mathrm{NAD})$ electrode at $37^{\circ} \mathrm{C}$ in $200 \mathrm{mM}$ potassium phosphate buffer $(\mathrm{pH}$

7.0) in the presence (black line) and absence (gray line) of $100 \mathrm{mM} \mathrm{L-proline} \mathrm{at} 10 \mathrm{mV} / \mathrm{s}$

Fig. 3 Power density curves of L-proline enzymatic biofuel cell (EFC) through enzyme cascade reaction.

L-Proline EFC was constructed using the eMcoP-immobilized electrode as a cathode, and Ap-LPDH 
265 Fig. 4 Long-term stability of L-proline EFC with eMcoP-immobilized cathode and 266 ApLPDH/ThP5CDH/PisGDH/GkNADHDH-immobilized anode. The relative load current was

267 defined as the percentage of the load current relative to the initial load current measured on the first

268 day of EFC fabrication

269

270 
Figures

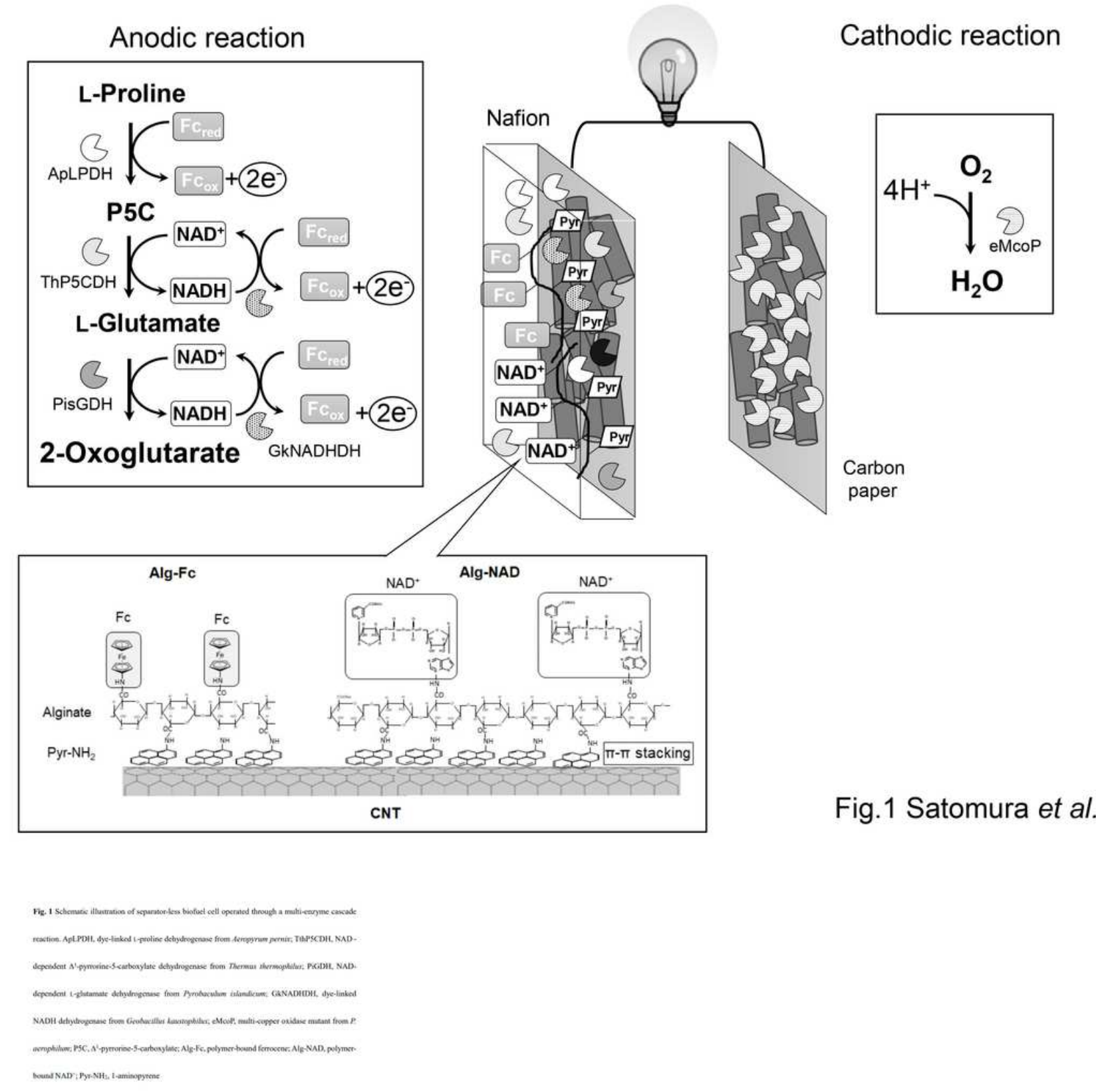

Figure 1

Please See image above for figure legend. 


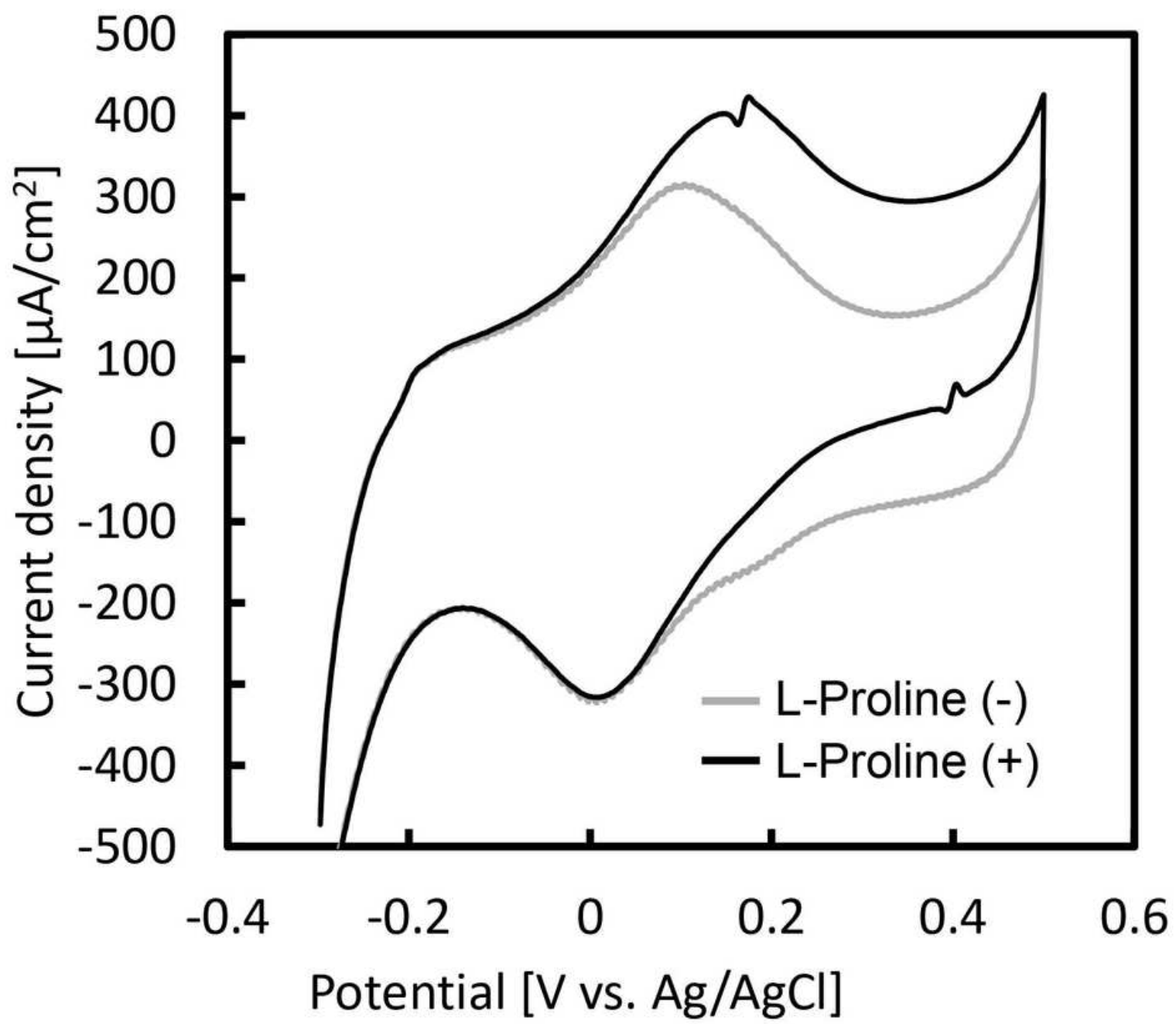

Fig. 2 Cyclic voltammetry curves of ApLPDH/ThP5CDH/PisGDH/GkNADHDH-modified $\mathrm{CP} / \mathrm{CNT} / \mathrm{Py}-\mathrm{NH}_{2}\left(-\mathrm{Alg}-\mathrm{Fc} /-\mathrm{Alg}-\mathrm{NAD}\right.$ ) electrode at $37^{\circ} \mathrm{C}$ in $200 \mathrm{mM}$ potassium phosphate buffer ( $\mathrm{pH}$ 7.0) in the presence (black line) and absence (gray line) of $100 \mathrm{mM} \mathrm{L-proline} \mathrm{at} 10 \mathrm{mV} / \mathrm{s}$

Figure 2

Please See image above for figure legend. 


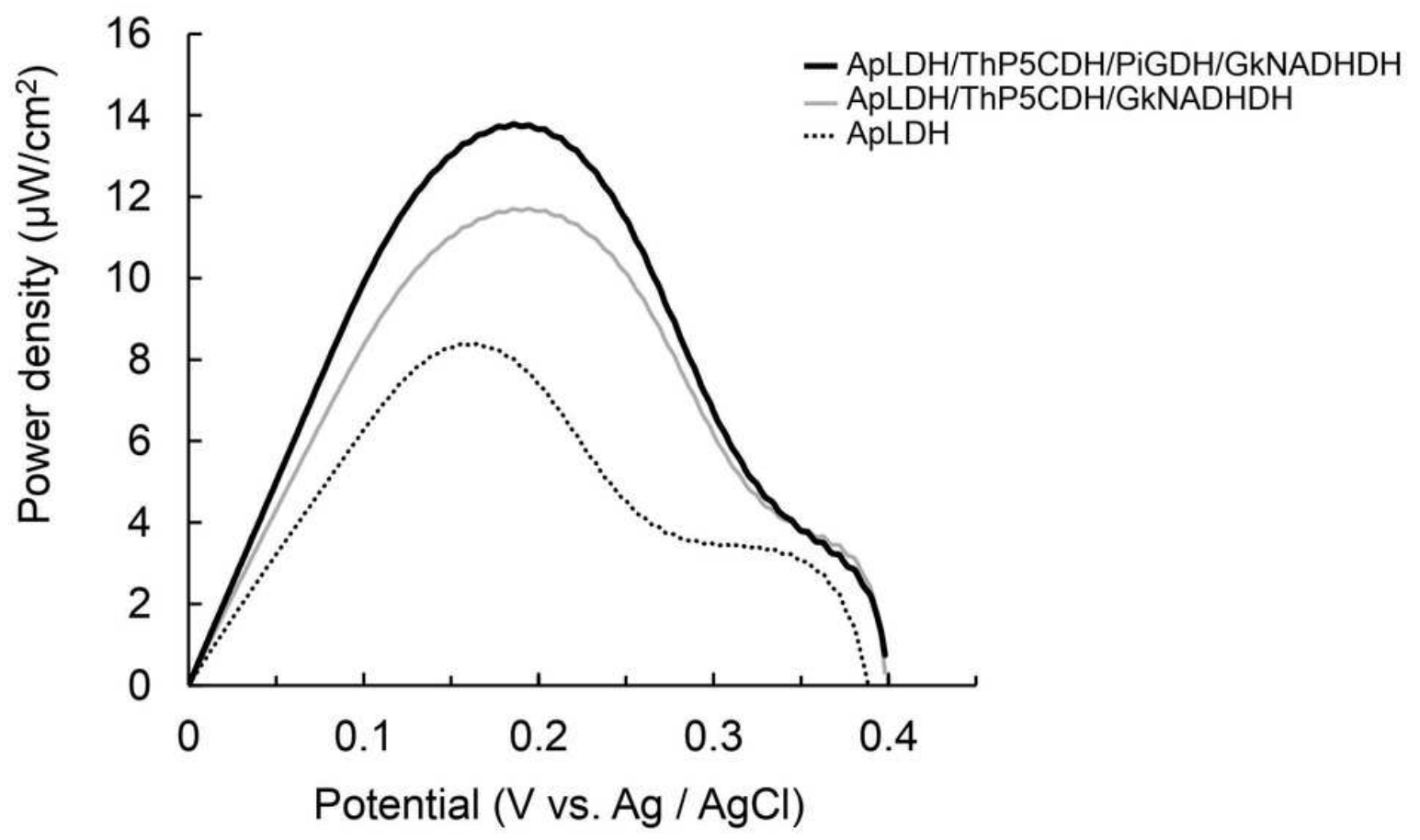

Fig. 3 Power density curves of L-proline enrymatic biofucl eell (EFC) through enzyme cascade

reaction.

1.Proline EFC was constructed using the CMcoP-immobilized clectrode as a cathode, and Ap-LPDH

(dotted line). Ap-LPDH/ThPSCDH/GkNADHDH (gray tine) of

APLPDHThPSCDH/PisGDH/GkNADHDH (black line) -modified electrode as the anode

Figure 3

Please See image above for figure legend. 


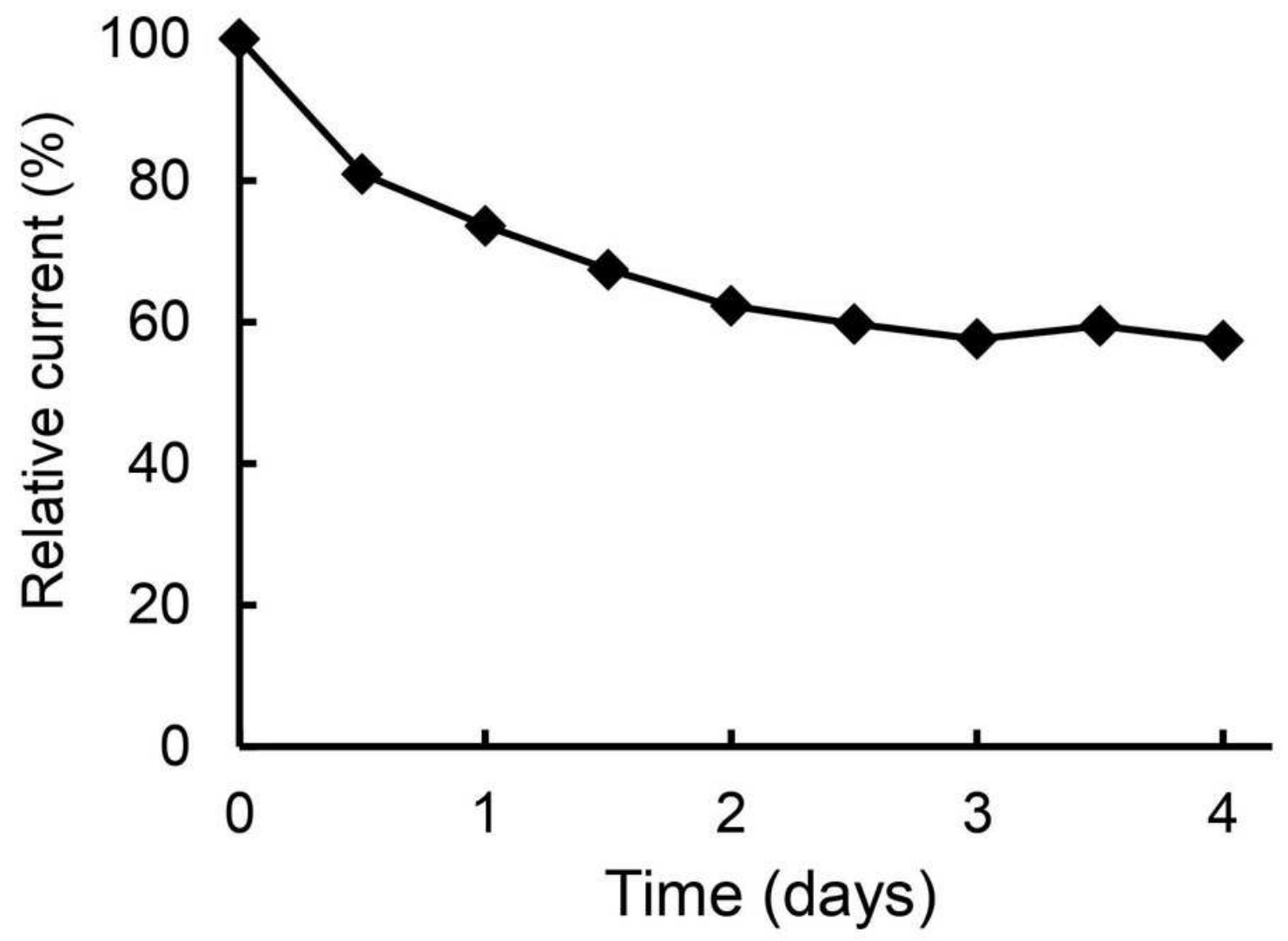

Fig. 4 Long-term stability of L-proline EFC with eMcoP-immobilized cathode and ApLPDH/ThP5CDH/PisGDH/GkNADHDH-immobilized anode. The relative load current was defined as the pereentage of the load current relative to the initial load current measured on the first day of EFC fabrication

Figure 4

Please See image above for figure legend. 